\title{
Instability of wake-dominated compressible mixing layers
}

\author{
Mei Zhuang \\ Department of Mechanical Engineering, Michigan State University, East Lansing, Michigan 48824 \\ Paul E. Dimotakis \\ Graduate Aeronautical Laboratories, California Institute of Technology, Pasadena, California 91125
}

(Received 26 July 1994; accepted 5 June 1995)

\begin{abstract}
The instability of supersonic mixing layers, with velocity profiles possessing a wake component, is investigated using linear, inviscid, spatial theory. The mean-velocity profile is represented by a hyperbolic-tangent profile plus a wake component. Such profiles are encountered in the initial region of experimental supersonic shear-layer flows, as well as in envisaged hypersonic propulsion systems in which ingested boundary layers generate substantial wake components. Shear-layer and wake instability modes previously found in incompressible mixing layers are also found in compressible mixing layers. The existence of a wake component in the velocity profile renders the mixing layer more unstable at all free-stream Mach numbers. For convective Mach numbers exceeding unity, the shear-layer mode splits into two supersonic modes, and the mixing layer becoming more unstable with increasing wake deficit. The wake mode becomes less unstable and eventually stable with increasing compressibility, i.e., increasing convective Mach numbers. (c) 1995 American Institute of Physics.
\end{abstract}

\section{INTRODUCTION}

Inviscid, spatial, linear hydrodynamic stability properties of shear-layer/wake flows have been studied in the past. In incompressible mixing layers with a wake component, the existence of an unstable wake mode was found in temporal stability calculations by Miksad. ${ }^{1}$ Koochesfahani and Frieler found two unstable modes, using spatial stability calculations, which they named the shear-layer mode and the wake mode. ${ }^{2}$ The shear-layer mode can be associated with the dynamics of the Kelvin-Helmholtz rollup. The rollup patterns of the wake mode resemble those seen in wakes. In the limit of unity velocity ratio, i.e., a symmetric wake in the absence of overall shear, the shear-layer, and wake modes approach the sinuous and varicose modes, respectively. ${ }^{3}$ For compressible, shear-layer/wake flows, the existence of the corresponding shear-layer and wake modes was investigated by Zhuang. ${ }^{4}$ In a stability study of laminar wakes, Lees and Gold showed that the sinuous mode is more unstable than the varicose mode. ${ }^{5}$ A strong stabilization effect of the Mach number on shear-layer/wake flows was demonstrated by Ragab in his linear-stability analysis. ${ }^{6}$ More recently, a stability study of developing supersonic mixing layers was conducted by Liang et al. ${ }^{7}$ in which three unstable modes were found.

Experimental results of compressible, unbounded, mixing layers indicate that mixing-layer growth basically stops decreasing, with increasing free-stream (and convective) Mach number, beyond a certain minimum value. ${ }^{8-10}$ In contrast, linear instability calculations of pure shear layers, i.e., mixing layers with monotonic mean-velocity profiles, suggest an ever-decreasing growth rate with increasing convective Mach number. ${ }^{11-13}$ Previous, two-dimensional, numerical simulations for compressible mixing layers, without consideration of the influence of a wake component, at least in the initial velocity profile, also showed a strong reduction in the mixing-layer growth rate as the free-stream convective
Mach number increases. ${ }^{14,15}$ In order to investigate possible mechanisms to explain the experimentally observed Machnumber behavior, the stability characteristics of compressible mixing layers are studied in this paper for a mean-velocity profile that includes a wake component.

It may be worth noting that in flows envisaged in hypersonic-propulsion devices, a mixing-layer zone will develop with substantial, if not dominant, wake components, as a consequence of the ingestion of very thick, turbulent boundary layers that have developed in the course of the large upstream fetch. As a consequence, not only is incorporating a strong wake component in the velocity profiles mandatory, but the classical descriptions in terms of the stability of monotonic, e.g., hyperbolic-tangent, error-function, or monotonic boundary-layer-type velocity profiles are also not directly applicable.

The investigations described in this paper are made of the case of inviscid flow, under the assumptions that the gases in the two streams are the same and the main flow can be treated as parallel. The disturbances to a mean mixinglayer flow with a wake (velocity-defect) component are assumed to be two or three dimensional (oblique), spatially developing, and of small amplitude.

\section{FORMULATION OF THE PROBLEM}

\section{A. The stability problem}

Consider a two-dimensional, parallel flow of two streams. With high-speed free-stream quantities as the reference and the local, transverse mixing-layer thickness as the length scale, the general dimensionless quantity of the flow field can be expressed as

$$
Q(x, y, t)=\bar{Q}(y)+Q^{\prime}(x, y, t)
$$

where $\bar{Q}(y)$ is the corresponding mean-flow quantity. The coordinates, $x$ and $y$, represent the dimensionless streamwise and transverse directions and $t$ the dimensionless time. As- 
sume that the flow is subjected to a two-dimensional, small disturbance, propagating in the $x$ direction, of the form

$$
Q^{\prime}(x, y, t)=f_{Q}(y) \exp [i \alpha(x-c t)],
$$

where $f_{Q}(y)$ is a dimensionless, complex eigenfunction of the $y$ coordinate, only; $\alpha$ is a dimensionless, complex wave number; and $c$ is a dimensionless, complex wave velocity.

From the basic disturbance equations for inviscid flows, a sccond-order differential equation can be derived for the dimensionless pressure-disturbance profile, $\pi(y)$, e.g., Ref. 4 ,

$$
\begin{aligned}
& \pi^{\prime \prime}(y)-\left(\frac{2 \bar{u}}{\bar{u}-c}-\frac{\bar{T}}{\bar{T}}\right) \pi^{\prime}(y)-\alpha^{2}\left(1-\frac{M_{1}^{2}}{\bar{T}}(\bar{u}-c)^{2}\right) \pi(y) \\
& -0 .
\end{aligned}
$$

In this expression, $M_{1}$ is the high-speed free-stream Mach number and $\bar{t}=\bar{t}(y)$ and $\bar{T}=\bar{T}(y)$ are the dimensionless mean-velocity and mean-temperature profiles, respectively.

The boundary conditions for the $\pi(y)$ eigenfunction are found by considering the limiting form of Eq. (3) far from the mixing layer, i.e., in the two free streams, as $y \rightarrow \pm \infty$. In that limit $\vec{u}^{\prime}(y)=\bar{T}^{\prime}(y)=0$, and the solutions to Eq. (3) are given by

$$
\pi(y)=A_{ \pm} \exp \left( \pm \lambda_{ \pm} y\right)
$$

where the $A_{ \pm}$are complex constants, and

$$
\begin{aligned}
& \lambda_{+}^{2}=\alpha^{2}\left[1-M_{1}^{2}(1-c)^{2}\right], \\
& \lambda_{-}^{2}=\alpha^{2}\left[1-M_{1}^{2}\left(u_{r}-c\right)^{2}\right] / T_{r},
\end{aligned}
$$

with $u_{r} \equiv u_{2} / u_{1}$ and $T_{r} \equiv T_{2} / T_{1}$ representing the mixing-layer free-stream velocity and temperature ratios, respectively.

The solution procedure is iterative. For a given disturbance (real) frequency, $\omega$, the complex eigenvalue, $\alpha$, is determined, such that the eigenfunction $\pi(y)$ of Eq. (3) satisfies the boundary conditions. A Runge-Kutta method, combined with a shooting technique, was used to solve this eigenvalue problem.

\section{B. The mean flow}

In most experimentally generated mixing layers, the initial region is dominated by the wake that arises from the finite thickness of the splitter plate and the drag of the two splitter-plate surfaces. The mean quantities of a developing shear-layer/wake flow, downstream of a splitter plate, can be obtained by direct numerical simulations. In order to simplify the analysis here, we assume that the mean-velocity profile is already known and composed of a hyperbolictangent profile plus a wake component that can be represented by a Gaussian, as was utilized by Koochesfahani and Frieler in their incompressible shear-layer/wake flow stability analysis. ${ }^{2}$ In this parametric model representation, the dimensionless mean-velocity profile can be written as

$$
\bar{l}(y)=u_{r}+\left(1-u_{r}\right) \zeta(y)-w e^{-\ln 2 y^{2}}
$$

where $u_{r}$ is the normalized velocity ratio, $w$ is the normalized wake deficit parameter, and

$$
\zeta(y) \equiv \frac{1}{2}[1+\tanh (y)] \text {. }
$$

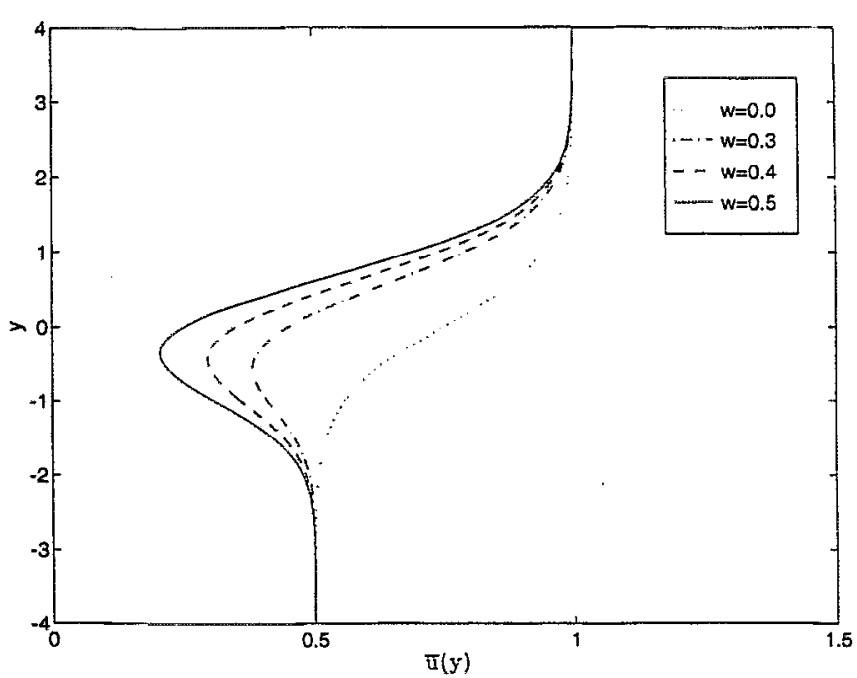

FIG. 1. Mean velocity profiles for a free-stream velocity ratio of $u_{r}=0.5$, for different values of the normalized wake deficit, $w$.

The mean-velocity profiles are plotted in Fig. 1, for a fixed velocity ratio, $u_{r}$, for different values of the wake deficit parameter, $w$. The dimensionless mean-temperature profiles were obtained using the Crocco-Busemann relation, ${ }^{16-18}$ and given by

$$
\bar{T}(y)=B_{1}+B_{2} \bar{u}(y)-\frac{y-1}{2} M_{1}^{2} \vec{i}(y) .
$$

In this expression, $B_{1}$ and $B_{2}$ are constants, as required to satisfy the free-stream boundary conditions on the temperature profile.

Mean-temperature profiles using this relation, for $M_{1}=3.0, T_{r}=1.0$, and $u_{r}=0.5$, are shown in Fig. 2 for different values of the wake deficit, $w$. The effect of a velocityprofilc wake component on the temperature profile can be seen to be considerable. The sensitivity of the results to the use of the Crocco-Busemann relation and the meantemperature profiles was assessed separately, and will be discussed below.

\section{RESULTS AND DISCUSSION}

Calculations of the instability characteristics of shearlayer/wake flows are discussed for two-dimensional, spatially developing disturbances, with a wake component given by $w=0.0,0.3,0.4$, and 0.5 , for a range of free-stream Mach numbers. The primary purpose of this work is to study the effect of a wake component on the instability behavior of compressible mixing layers. As a consequence, the velocity and temperature ratios were fixed at $u_{r}=0.5$ and $T_{r}=1.0$ in all the calculations.

The convective Mach number has been suggested as the appropriate parameter scaling the effects of compressibility in supersonic shear layer flows. ${ }^{9,19}$ In this calculation, it is defined, separately for each free stream, as 


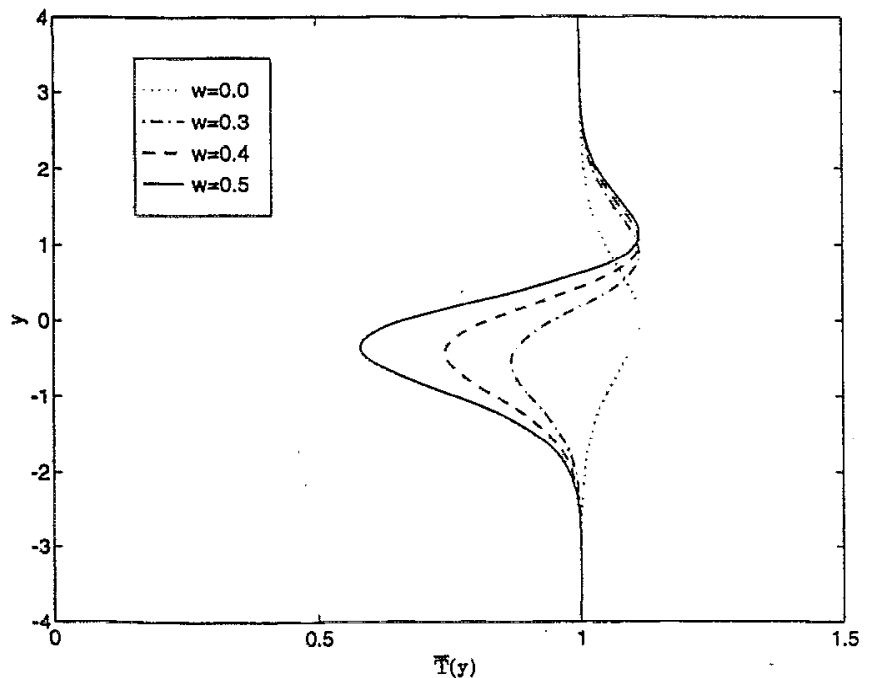

FIG. 2. Mean temperature profiles for a free-stream velocity ratio $u_{r}=0.5$, temperature ratio $T_{r}=1.0$, and a high-speed Mach number of $M_{1}=3.0$, for different values of the normalized wake deficit, $w$.

$$
M_{c 1}=\frac{u_{1}-c_{p}}{a_{1}}, \quad M_{c 2}=\frac{c_{p}-u_{2}}{a_{2}},
$$

where $a_{1}$ and $a_{2}$ are the free-stream speeds of sound and $c_{p}$ is the phase velocity of the most unstable eigenvalue. ${ }^{\text {" }} \mathrm{Al}$ ternative definitions of the convective Mach number have also been given, in which other estimates of the convective velocity, $u_{c}$, of the large-scale structures were used instead. ${ }^{9,12,19,20}$ We regard the definition given by Eq. (9) as the appropriate one, in the context of the present discussion, providing a self-consistent definition with unstable-mode behavior, as it relies on the phase velocity of the disturbances that is derived from the stability analysis itself.

The spatial instability characteristics of the shear-layer/ wake flow, as a function of the normalized wake deficit, $w$, are shown in Figs. 3(a) and 3(b), for subsonic convective Mach numbers, i.e., $M_{c 1}<1$ and $M_{c 2}<1$. One of the main results, shown in Figs. 3(a) and 3(b), is that, for nonzero values of the wake parameter, $w$, subsonic disturbances possess two unstable modes. These two unstable modes were classified as the shear-layer mode and the wake mode by Koochesfahani and Frieler. ${ }^{2}$ In the zero-wake-component limit, the shear-layer mode approaches the solution for the hyperbolic-tangent mean-velocity-profile solution, while the wake mode vanishes. Another feature is also apparent in these figures. The neutral points of the shear-layer mode and the wake mode move to lower frequencies, while the maximum amplification rates of the modes increase as the wake parameter, $w$, increases. This result is consistent with previous studies for incompressible of shear/wake mixing-layer flows.

After the disturbances of the shear-layer mode become supersonic, the shear-layer mode splits into two supersonic modes, whereas the wake mode remains unsplit (cf. Fig. 4). Therefore, three unstable modes are found. These findings agree with those of Liang et al. ${ }^{7}$ The wake mode in our studies is related to the slow Mode II in the work of Liang et al. ${ }^{7}$

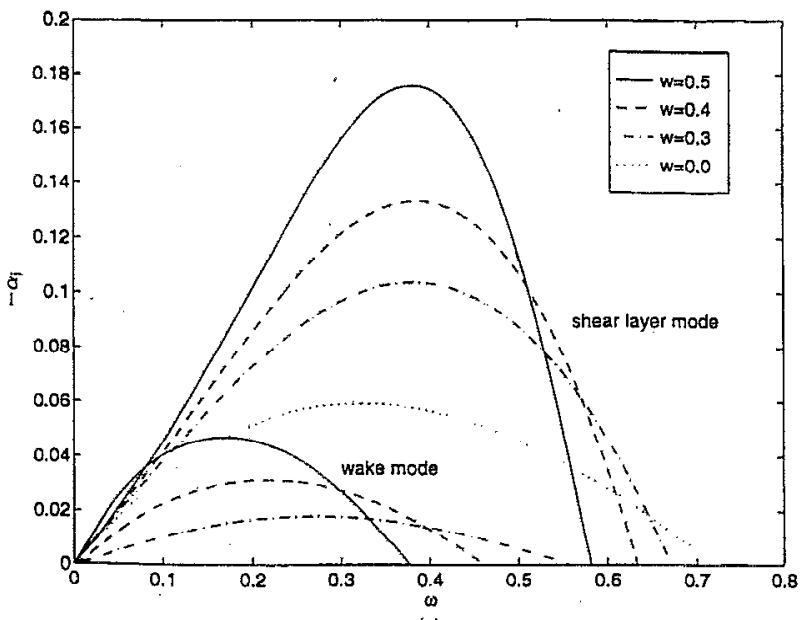

(a)

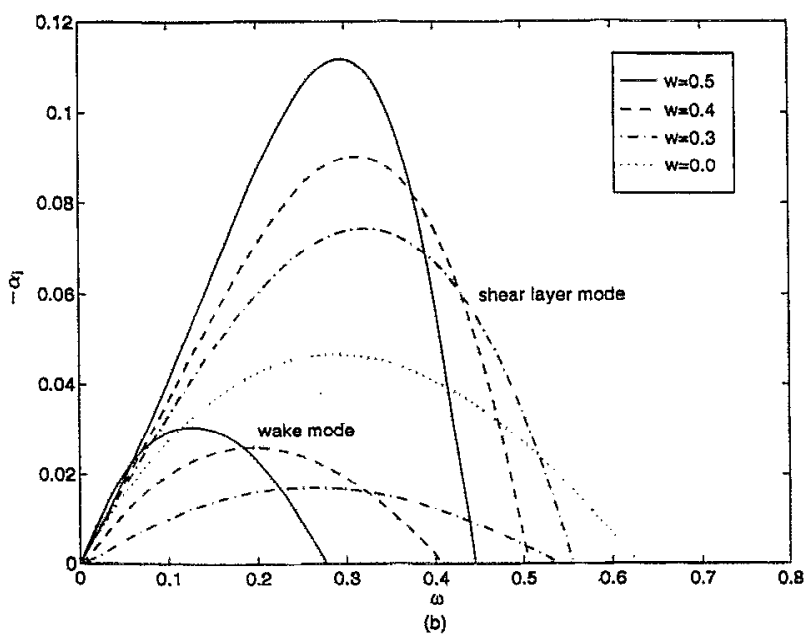

FIG. 3. Instability characteristics for $u_{r}=0.5$ and $T_{r}=1.0$. (a) $M_{1}=1.0$; (b) $M_{1}=2.0$.

The mode-splitting phenomenon was first identified by Jackson and Grosch, ${ }^{20}$ in supersonic mixing layers without a wake component. They classified the two supersonic modes as a slow supersonic mode, i.e., supersonic relative to the high-speed stream, and a fast supersonic mode, i.e., supersonic relative to the low-speed stream. Here we define the more unstable supersonic shear mode as the strong mode and the less unstable supersonic shear mode as the weak mode. In the calculation of Liang et al. ${ }^{7}$ the slow Mode II (wake mode) was the dominant mode in the initial mixing zone and became stable in the downstream region. In contrast to their result, the slow supersonic shear-layer mode emerges as the dominant mode in our investigations. We are not totally surprised by this difference, since which of the supersonic modes is more unstable can be expected to depend on the free-stream and mean-flow conditions. The work of Koochesfahani and Frieler, on incompressible shear-layer/ wake flows, also showed a similar behavior, i.e., a dominant shear-layer mode, for some mean-flow conditions, and a dominant wake mode for others. As can be also seen in Fig. 4 , the existence of a wake component can render the strong supersonic shear mode more unstable and the weak supersonic shear mode less unstable. 

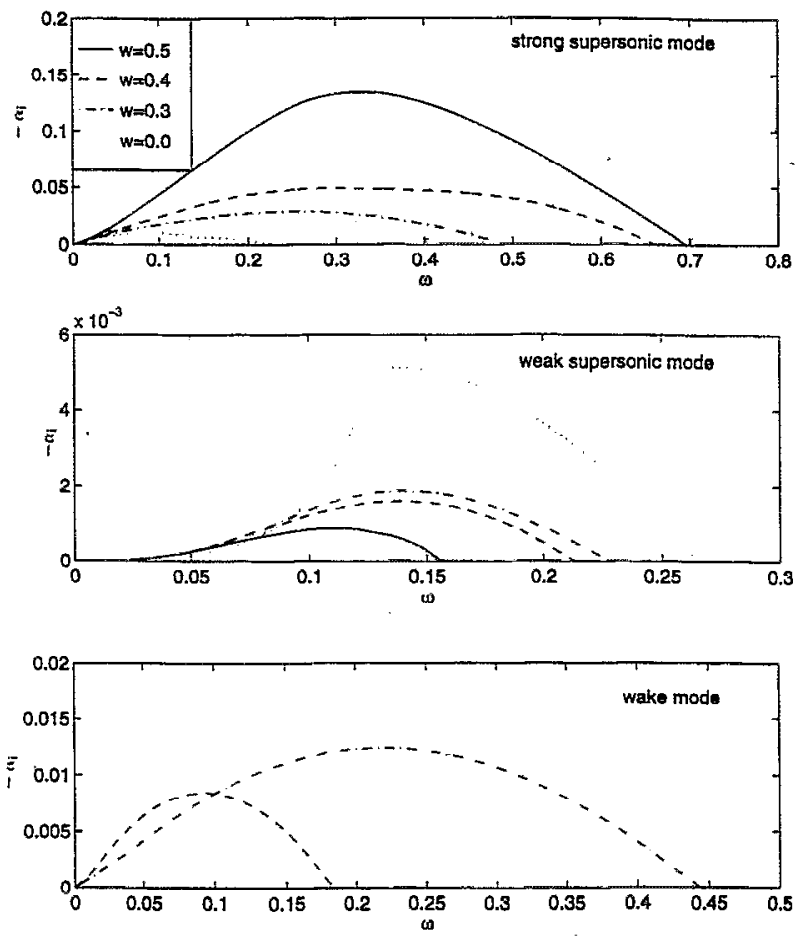

FIG. 4. Effect of the wake deficit, $w$, on the instability characteristics of $u_{r}=0.5, T_{r}=1.0$, and $M_{1}=4.0$ mixing layers. The wake mode is absent for $w=0.0$ and stable for $w=0.5$, and not shown.

As the free-stream Mach number increases to a supersonic convective Mach number regime, the maximum amplification rate of the strong supersonic shear-layer mode increases to its local extremum value and then slightly falls again, while the weak supersonic shear mode and the wake mode eventually become stable [cf. Figs. 5(a)-5(c)]. Figure 5(c) shows that although the maximum amplification rate of the wake mode increases with increasing wake parameter, $w$, at the lower free-stream Mach numbers, at higher values of the wake parameter, the wake mode is the first to become stable as the free-stream Mach number increases.

There is evidence in support of the proposal that experimental mixing-layer growth rates can be modeled as proportional to the maximum amplification rate of the unstable disturbances (e.g., Refs. 11, 15, 20, and 21). The result depicted in Fig. 5(a) shows that a wake component can be responsible for higher growth rates for supersonic mixing layers.

Linear-stability analysis suggests that oblique waves will tend to dominate (unbounded) mixing layers with monotonic mean-velocity profiles at high Mach numbers. ${ }^{4,15,20-22}$ Our calculations indicate that this is not always true for supersonic, shear/wake mixing-layer flows. Oblique-wave mode calculations are plotted in Figs. 6(a) and 6(b), for wakeparameter values of $w=0.3$ and 0.5 . It can be seen that the two-dimensional mode is the dominant mode, if a strong wake component exists in the mean-velocity profile. Conversely, a relatively weak wake component corresponds to a flow in which oblique waves dominate.

Stability studies of wake-free shear layers inside a rectangular channel, hy Tam and Hu, as well as others, showed that two-dimensional, supersonic, instability waves are char-
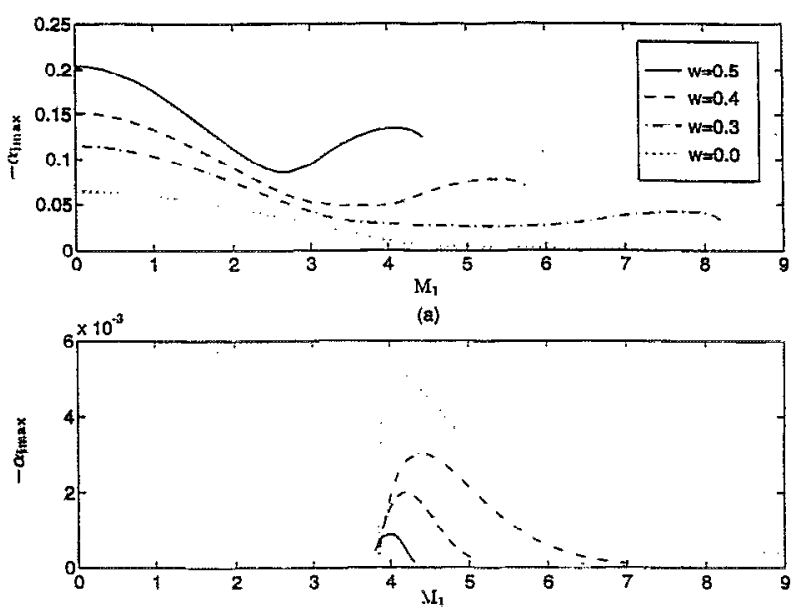

(b)

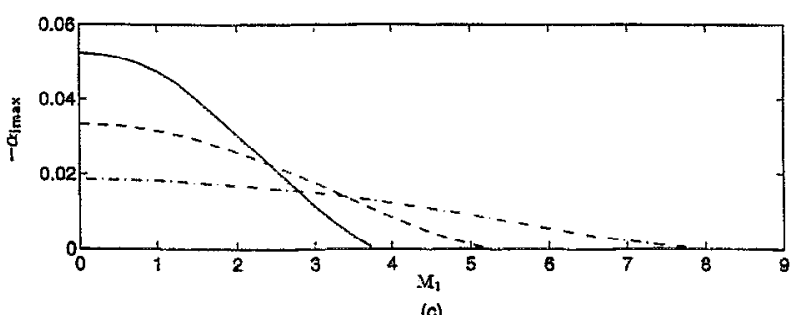

(c)

FIG. 5. Maximum amplification rates for the shear-layer and wake modes for $u_{r}=0.5, T_{r}=1.0$, versus free-stream Mach number, $M_{1}$, for different values of $w$. (a) Shear-layer mode (supersonic strong mode for supersonic disturbances); (b) supersonic weak mode; (c) wake mode.

acterized by larger spatial growth rates than their threedimensional (oblique) counterparts, for reasonably thick shear layers. 23,24

Liang et al. ${ }^{7}$ showed that the two-dimensional mode was the dominant mode for some downstream locations $\left(x^{\prime}=0.05,0.1\right.$, and 0.5). The downstream locations they chose were still in the initial mixing zone, however, and

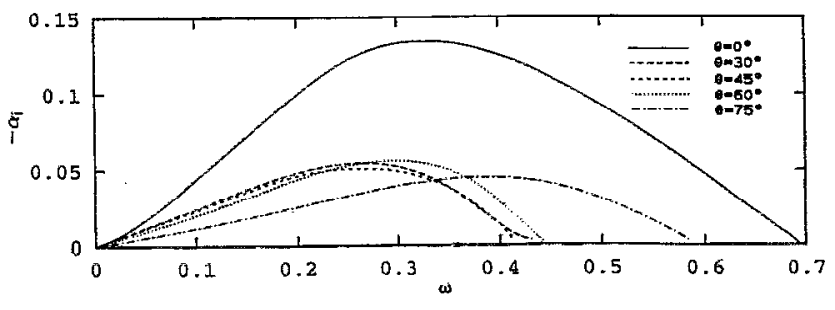

(a)

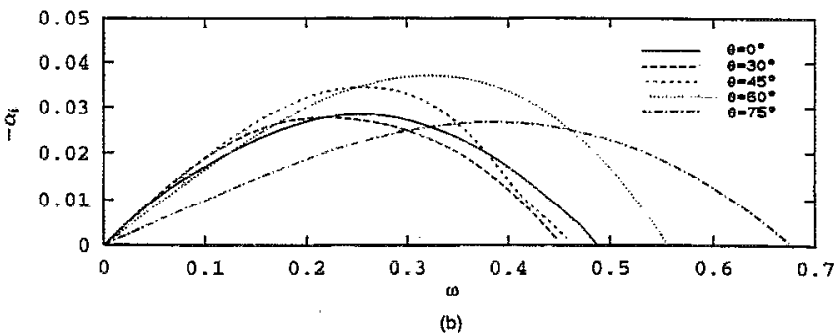

FIG. 6. Amplification rate for three-dimensiunal disturbances: $u_{r}=0.5$, $T_{r}=1.0$, and $M_{1}=4.0$. (a) $w=0.5$; (b) $w=0.3$. 
characterized by relatively strong wake components (cf. mean-velocity profile plot in their paper). Our result is in agreement with theirs, i.e., that the two-dimensional mode is the most unstable one for a shear-layer/wake flow with a strong wake component. They did not calculate obliquewave behavior at farther downstream locations, such as $x^{\prime}=1.0,2.0, \ldots$, etc., however, and we cannot make any direct comparisons. But the fact that they found the oblique mode to be the dominant one, in the case of a small wake component, indirectly confirms our findings for supersonic mixing layers with relatively small wake components.

The pressure eigenfunctions, $\pi(y)$, corresponding to the most amplified shear-layer (the supersonic strong mode, if the disturbances are supersonic) and wake modes are plotted in Figs. 7(a)-7(d), for free-stream Mach numbers, $M_{1}=1,2$, 3 , and 4 , and a wake parameter of $w=0.4$. The solid lines in these figures depict the real part and the dashed lines the imaginary part of the complex pressure eigenfunctions, $\pi(y)$ $=p(y)+i q(y)$. For the case of $M_{1}=1.0[$ Fig. 7(a)], the real and imaginary parts of both modes decay exponentially away from the mixing layers, indicating subsonic disturbances. The calculations of the convective Mach numbers of these two modes also indicate subsonic disturbances (for the shearlayer mode $M_{c 1}=0.36$ and for the wake mode $M_{c 1}=0.62$ ). As the free-stream Mach number increases, the wake mode disturbances are the first to become supersonic on the highspeed side [cf. Fig 7(b): $M_{c 1}=0.71$ for the shear-layer mode, while $M_{c 1}=1.26$ for the wake mode]. The radiative nature of the supersonic instability waves of the wake mode is evident in Fig 7(b). The pressure eigenfunctions, $\pi(y)$, of the supersonic strong mode and the wake mode are shown in Figs. $7(\mathrm{c})$ and $7(\mathrm{~d})$, for $M_{1}=3\left(M_{c 1}=1.19\right.$ for the supersonic strong mode, while $M_{c l}=1.91$ for the wake mode) and $M_{1}=4$, respectively. The pressure eigenfunctions, $\pi(y)$, display an oscillatory decay in the stream with supersonic disturbances and an exponential decay in the stream with subsonic disturbances.

As noted above, the convective Mach number was based on the computed phase velocity, $c_{p}$, of the most unstable disturbance, instead of an externally defined convective velocity, $u_{c}$. If the convective velocity, $u_{c}$, is estimated as that of the frame in which the isentropically recovered total pressure is matched, ${ }^{9}$ for example, we would get a result indicating that mixing-layer disturbances, shown in Fig. 7(c), are subsonic, even though the (radiative) nature of supersonic disturbances indicates otherwise. Such estimates for the convection velocity, $u_{c}$, cannot be used to calculate the convective Mach numbers for mixing layers with a wake component.

The calculations were carried out to values of the freestream Mach number of $M_{1}-4.5,5.75$, and 8.0; for values of the wake deficit of $w=0.5,0.4$, and 0.3 [cf. Figs. 5(a) -5 (c)], respectively, to avoid local values of the mean-temperature profile, $\bar{T}(y)$, that become too small, and, eventually, negative (by virtue of the Crocco-Busemann relation). The mean-temperature profiles, $\bar{T}(y)$, and the local Mach-number profiles, $M(y)$, where the calculations were stopped, are plotted in Fig. 8.

As noted above, mean-temperature profiles were derived
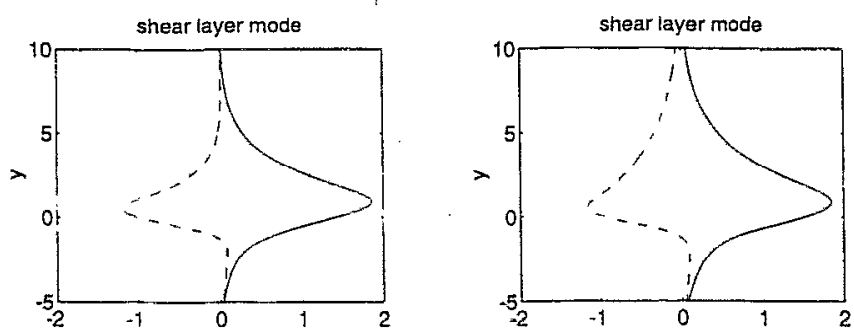

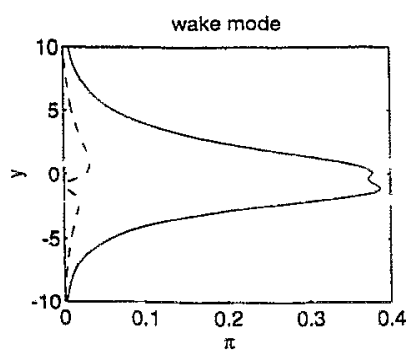

(a)
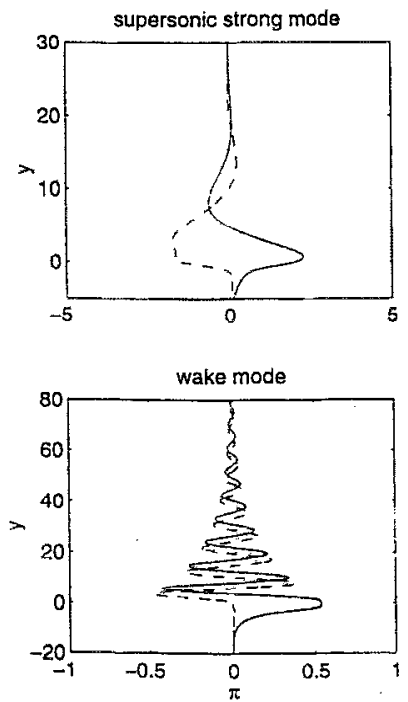

(c)

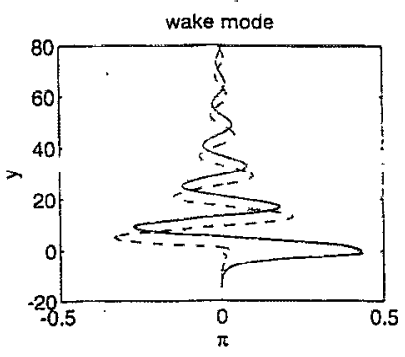

(b)
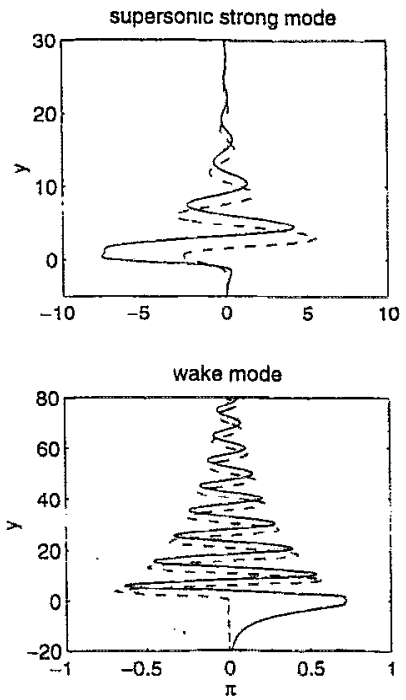

(d)
FIG. 7. Pressure eigenfunctions, $\pi(y)=p(y)+i q(y)$, of the most amplified modes for mixing layers, with $u_{r}=0.5, T_{r}=1.0$, and $w=0.4 . p(y):-, q(y)$ : ... (a) $M_{1}=1.0$, (b) $M_{1}=2.0$, (c) $M_{1}=3.0$, and (d) $M_{1}=4.0$.

from the Crocco-Busemann relation, which represents an independent assumption in these calculations. Specifically, on the assumption that the mean-temperature profile, $\bar{T}(y)$, depends on the mean profile, $\bar{u}(y)$, of the streamwise velocity component, and that the Prandtl number is equal to unity. Variations in the specific heat and Prandtl number with temperature were not taken into account. If the local temperature becomes too small, the local Mach number would be extremely high and the mixing layer becomes hypersonic. At such high Mach numbers, variations in the specific heat and Prandtl number with temperature are no longer negligible, and these assumptions can be expected to fail.

In order to check the sensitivity of our results on the assumptions that produced the mean-temperature profiles, two alternative mean-temperature profiles were also considered. The first alternative was represented by a hyperbolictangent profile plus a wake component, i.e., 

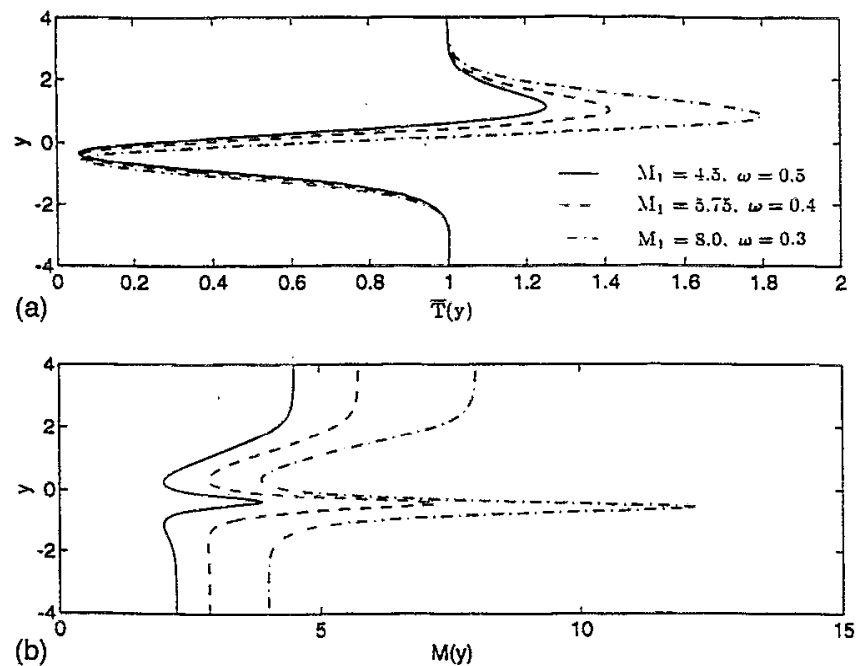

FIG. 8. Mixing-layer behavior for $u_{r}=0.5$ and $T_{r}=1.0$. (a) Temperature profiles for $M_{1}=4.5, w=0.5 ; M_{1}=5.75, w=0.4 ;$ and $M_{1}=8.0, w=3.0$. (b) Local Mach number profiles for the three cases.

$$
\bar{T}(y)=T_{r}+\left(1-T_{r}\right) \zeta(y)-w_{T} e^{-\ln 2 y^{2}} .
$$

The second alternative was a hyperbolic-tangent profile only $\left(w_{T}=0\right)$, i.e.,

$$
\bar{T}(y)=T_{r}+\left(1-T_{r}\right) \zeta(y) .
$$

Comparisons of these two mean-temperature profiles with the Crocco-Busemann relation are shown in Fig. 9, for $u_{r}=0.5, T_{r}=1.0$, and $w=0.4$, at different free-stream Mach numbers. The maximum amplification rate versus freestream Mach number, $M_{1}$, is plotted in Fig. 10(a) for the three mean-temperature profiles, using the same meanvelocity profile with a wake component. For subsonic convective Mach numbers, which correspond to the range $M_{1}<3$ for these flow parameters, nearly identical maximum
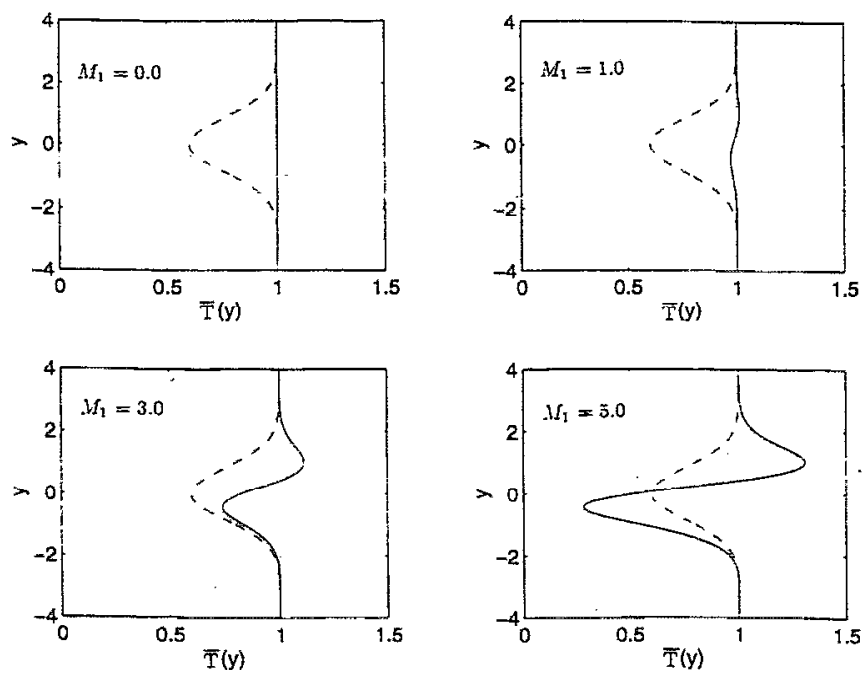

FIG. 9. Mean-temperature profiles for $u_{r}=0.5$ and $T_{r}=1.0$. CroccoBusemann relation with $w=0.4: \quad ;$ hyperbolic-tangent temperature profile with $w=0.4$ : ---; Hyperbolic-tangent temperature profile with $w=0.0: \cdots$.

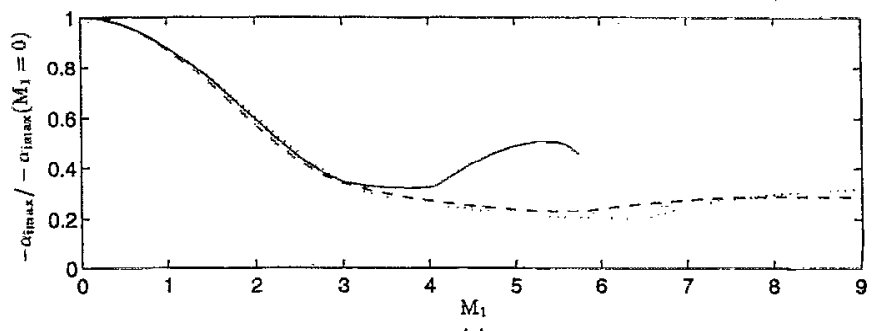

(a)

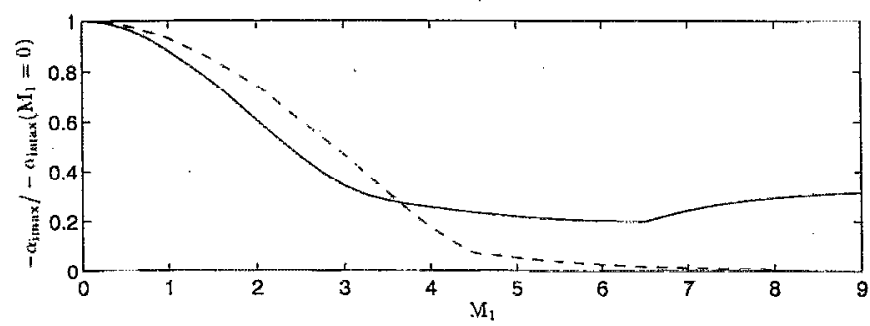

(b)

FIG. 10. Normalized amplification rates of the shear-layer mode of the velocity profile with $w=0.4$ (supersonic strong mode for supersonic disturbances), versus the free-stream Mach number, $M_{1}$, for $u_{r}=0.5$ and $T_{r}=1.0$. (a) Crocco-Busemann relation with $w=0.4:-$; hyperbolic-tangent temperature profile with $w=0.4:-\cdots$; hyperbolic-tangent temperature profile with $w=0.0: \cdots$, (b) Hyperbolic-tangent temperature profile with $w=0.0$; velocity profile with $w=0.4$ : —; velocity profile with $w=0.0$ : ---.

amplification rates are obtained with the Crocco-Busemann relation and the hyperbolic-tangent mean-temperature profile of Eq. (11). The maximum amplification rate is higher, however, when the hyperbolic-tangent temperature profile plus a wake component [i.e., Eq. (10)] is used. The difference between the maximum amplification rate decreases as the freestream Mach number increases. In the supersonic convective Mach number regime, similar trends in the maximum amplification rate are observed, regardless of the meantemperature profile used. Figure 10(b) shows the calculated maximum amplification rate using the same hyperbolictangent temperature profile, withoul a wake component [Eq. (11)], and two different velocity profiles, with and without a wake component. For the case with no wake component, the maximum amplification rate decreases monotonically to zero as the free-stream Mach number increases into the supersonic region, as has been noted in previous studies, ${ }^{4,15,20,21}$ in clear contrast, as shown here, to flows with a wake component in the velocity profile.

The data in Figs. 10(a) and 10(b) are replotted in Figs. 11 (a) and $1 \mathrm{l}(\mathrm{b})$, versus the high-speed free-stream convective Mach number, $M_{c 1}$, computed on the basis of the phase velocity, $c_{p}[\mathrm{Fq} .(9)$. In terms of this variable, the maximum amplification rate reflects the change in behavior at a convective Mach number of unity, as do the experimental mixing-layer growth data, and lends additional support for the choice made in Eq. (9) as the definition of the convective Mach number(s), as the appropriate one for this analysis.

For supersonic mixing layers, we may conclude, on the basis of the results depictcd in Figs. 10 and 11, that the mean-temperature profile has a weaker effect on the maximum amplification rate. It is the presence of a wake component in the velocity profile that exerts the crucial influence on 


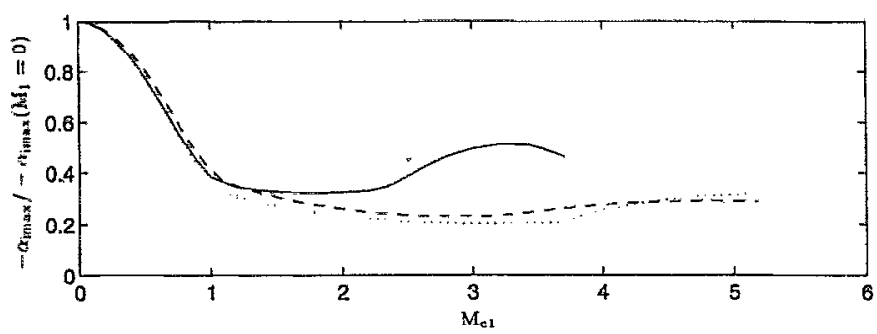

(a)

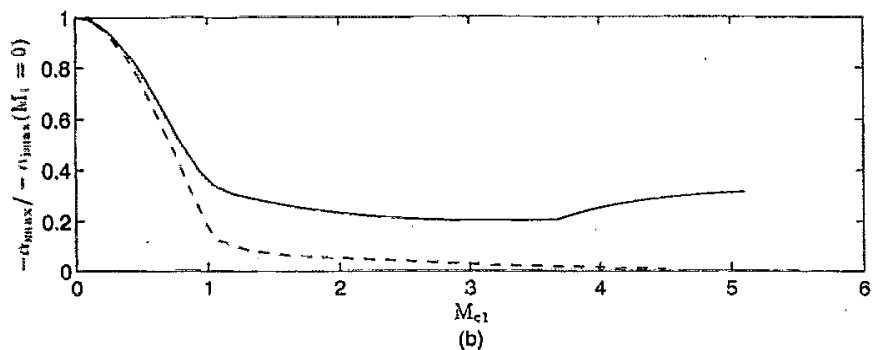

FIG. 11. Normalized amplification rates of the shear-layer mode versus the convective Mach number, $M_{c 1}$. For the legend, see Fig. 10 .

the growth rate. Supersonic mixing layers with a wake component in the mean-velocity profile become more unstable and are characterized by a maximum amplification rate that stops decreasing with increasing free-stream Mach number.

\section{CONCLUSIONS}

Our results show that the existence of a wake component in the mean-velocity profile of compressible mixing layers can have a large affect in the stability behavior of the flow. In incompressible shear/wake mixing-layer flows, there are two unstable modes, the shear-layer mode and the wake mode. The maximum amplification rates of these two modes increase with increasing wake deficit. For supersonic convective Mach numbers, the shear-layer mode splits into two supersonic modes, the strong supersonic mode and the weak supersonic mode, whereas the wake mode remains unsplit. In supersonic mixing layers, two-dimensional modes are dominant, if the mean-velocity profile is characterized by a strong wake component. With a relatively small, or no, wake component, oblique wave modes (in unbounded shear layers) are dominant. Studies of wake-dominated mixing layers also suggest that larger values of the amplification rate at high free-stream Mach numbers can be obtained by increasing the wake deficit. For supersonic mixing layers with a wake component in the mean-velocity profile, the mixing layer becomes more unstable and the maximum amplification rate does not decrease monotonically to zero with increasing freestream Mach number.

\section{ACKNOWLEDGMENTS}

We would like to acknowledge our discussions with Professor T. Kubota and many helpful comments by Professor
M. Koochesfahani and M. Slessor. This work was supported by the Air Force Office of Scientific Research, Grant Nos. 88-0155 and F49620-94-1-0353.

'R. W. Miksad, "Experiments on the nonlinear stages of free shear-layer transition," J, Fluid Mech. 56, 695 (1972).

${ }^{2} \mathrm{M}$. M. Koochesfahani and C. E. Frieler, "Instability of nonuniform density free shear layers with a wake profile," AIAA J. 27, 1735 (1989).

${ }^{3}$ G. E. Mattingly and W. O. Criminale, "The stability of an incompressible two-dimensional wake," J. Fluid Mech. 51, 233 (1972).

${ }^{4} \mathrm{M}$. Zhuang, "An investigation of the inviscid spatial instability of compressible mixing layers," $\mathrm{Ph} . \mathrm{D}$. thesis, California Institute of Technology, 1990.

${ }^{5}$ L. Lees and H. Gold, "Stability of laminar boundary layers and wakes at hypersonic speeds. Part I. Stability of laminar wakes," Proceedings of the International Symposium on Fundamental Phenomena in Hypersonic Flows (Cornell University Press, Ithaca, 1966), p. 310.

${ }^{6} \mathrm{~S}$. A. Ragab, "Instabilities in the wake/mixing-layer region of a splitter plate separating two supersonic streams," Proceedings of the lst National Fluid Dynamics Congress 1988, Vol. II, pp. 1095-1102.

${ }^{7}$ F. Liang, E. Reshotko, and A. Demetriades, "A stability study of the developing mixing layer formed by two supersonic laminar streams," AIAA Paper No. 93-0345, 1993; also submitted to Phys. Fluids.

${ }^{8}$ N. Chinzei, G. Masua, T. Komuro, A. Murakami, and K. Kudou, "Spreading of two-stream supersonic turbulent mixing layers," Phys. Fluids 29, 1345 (1986).

'D. Papamoschou and A. Roshko, "The compressible turbulent shear layer: An experimental study," J. Fluid Mech. 197, 453 (1988).

${ }^{10}$ J. L. Hall, P. E. Dimotakis, and H. Rosemann, "Experiments in nonreacting compressible shear layers," AIAA J. 31, 2247 (1993).

${ }^{11}$ M. Zhuang, T. Kubota, and P. E. Dimotakis, "On the stability of inviscid, compressible free shcar layers," in Ref. 6, pp. 768-773; also, "Instability of inviscid, compressible tree shear layers," AIAA J. 28, 1728 (1990).

${ }^{12}$ S. A. Ragab and J. L. Wu, "Linear instabilities in two-dimensional compressible mixing layers," Phys. Fluids A 1, 957 (1989).

${ }^{13}$ N. D. Sandham and W. C. Reynolds, "Compressible mixing layer: Linear theory and direct simulation," AIAA J. 28, 618 (1990).

${ }^{14} \mathrm{~S}$. K. Lele, "Direct numerical simulation of compressible free shear flows," AIAA 27th Aerospace Sciences Meeting, Paper No. 89-0374, 1989.

${ }^{15}$ N. D. Sandham and W. C. Reynolds, "A numerical investigation of the compressible mixing layer," Stanford Report No. TF-45, 1989.

${ }^{16} \mathrm{~L}$. Crocco, "Sulla transmissione del calore da una lamina piana a un fluid scorrente ad alta velocita," L' Aerotecnica 12, 181 (1932).

${ }^{17} \mathrm{~A}$. Busemann, "Gasströmung mit laminarer grenzschicht entlang einer platte," Z. Angew Math. Mech. 15, 23 (1935).

${ }^{18} \mathrm{H}$. Schlichting, Boundary-Layer Theory, 6th ed. (McGraw-Hill, New York, 1968).

${ }^{19}$ D. W. Bogdanoff, "Compressibility effects in turbulent shear layers," AIAA J. 21, 926 (1983).

${ }^{20}$ T. L. Jackson and C. E. Giosch, "Inviscid spatial stability of a compressible mixing layer," J. Fluid Mech. 208, 609 (1989).

${ }^{21}$ S. A. Ragab and J. L. Wu, "Instabilities in the free shear layer formed by two supersonic streams," AIAA 26th Aerospace Sciences Meeting, Paper No. 88-0038, 1988.

${ }^{22} \mathrm{H}$. Gropengiesser, "Study of the stability of boundary layers in compressible fluids," NASA-TT-F-12786, 1970.

${ }^{23} \mathrm{C}$. K. W. Tam and F. Q. Hu, "The instability and acoustic wave modes of supersonic mixing layers inside a rectangular channel," J. Fluid Mech. 203, 51 (1989).

${ }^{24} \mathrm{M}$. Zhuang, P. E. Dimotakis, and T. Kubota, "The effect of walls on a spatially growing supersonic shear layer," Phys. Fluids A 2, 599 (1990). 